\title{
Using pipe-in-pipe systems for subsea pipeline vibration control
}

\author{
Kaiming $\mathrm{Bi}^{*}$, Hong Hao \\ Centre for Infrastructural Monitoring and Protection, School of Civil and Mechanical \\ Engineering, Curtin University, Kent Street, Bentley WA 6102, Australia
}

\begin{abstract}
Pipe-in-pipe (PIP) systems are increasingly used in subsea pipeline applications due to their favourable thermal insulation capacity. Pipe-in-pipe systems consist of concentric inner and outer pipes, the inner pipe carries hydrocarbons and the outer pipe provides mechanical protection to withstand the external hydrostatic pressure. The annulus between the inner and outer pipes is either empty or filled with non-structural insulation material. Due to the special structural layout, optimized springs and dashpots can be installed in the annulus and the system can be made as a structure-tuned mass damper (TMD) system, which therefore has the potential to mitigate the pipeline vibrations induced by various sources. This paper proposes using pipe-in-pipe systems for the subsea pipeline vibration control. The simplification of the pipe-in-pipe system as a non-conventional structure-TMD system is firstly presented. The effectiveness of using pipe-in-pipe system to mitigate seismic induced vibration of a subsea pipeline with a free span is investigated through numerical simulations by examining the seismic responses of both the traditional and proposed pipe-in-pipe systems based on the detailed three dimensional (3D) numerical analyses. Two possible design options and the robustness of the proposed system for the pipeline vibration control are discussed. Numerical results show that the proposed pipe-in-pipe system can effectively suppress seismic induced vibrations of subsea pipelines without changing too much of the traditional design. Therefore it could be a cost-effective solution to mitigate pipe vibrations subjected to external dynamic loadings.
\end{abstract}

Keywords: pipe-in-pipe system, subsea pipeline, vibration control, TMD

*Corresponding author. Tel: +61 89266 5139; fax: +61 892662681

E-mail address: kaiming.bi@curtin.edu.au (K. Bi) 


\section{Introduction}

Pipe-in-pipe (PIP) systems are increasingly used in subsea pipeline applications due to the exceptional level of thermal insulation they provide. Pipe-in-pipe systems consist of an inner pipe, conveying the hydrocarbons, and an outer pipe, withstanding the external hydrostatic pressure. The annulus between the inner and outer pipes is either empty or filled with nonstructural insulation material like mineral wool, polyurethane foam or aerogel [1]. Thanks to their exceptional thermal insulation capacity, pipe-in-pipe systems are well suited for the transportation of hydrocarbons at high pressure and high temperature (HP/HT), preventing hydrate formation and ensuring high discharge temperature at the arrival facility. Today, pipe-in-pipe systems are widely used in the North Sea, the Pacific, Gulf of Mexico and Africa.

Previous studies on subsea pipe-in-pipe systems mainly focused on the structural instabilities. For example, extensive experimental and numerical investigations have been carried out on the propagation buckling (e.g. [2-5]) and upheaval buckling [6] phenomena of subsea pipe-in-pipe systems. Besides these buckling issues, another factor that may severely threaten the integrity of subsea pipelines is the vibrations of free spans induced by various sources such as vortex shedding or earthquake. It is known that free spans can be formed due to the seabed irregularities during installation or the subsequent scouring and pipeline horizontal movements during operation [1]. Pipeline free spans can have a critical influence on the safety and integrity of the pipeline operation since they are susceptible to vortexinduced vibrations (VIV) and hence fatigue damage. Moreover, subsea pipelines may traverse through seismic active zones, different seismic hazards may impose severe damages to the pipeline systems. A review of many previous earthquake events reveals that for the buried pipelines, the permanent ground deformation due to soil failure may have severe influence on the pipeline integrity [7]. While for the unburied pipelines, both seismic ground waves and permanent ground deformation can cause severe damage to the pipelines [1].

Vortex shedding induced vibrations on the subsea pipelines have been systematically studied by many researchers and various vibration control methods and devices have been developed (e.g. [8, 9]). Kumar et al. [10] provides an excellent review on these methods. For the seismic responses of subsea pipelines, literature review reveals that previous studies are rare. Nath and Soh [11] investigated the harmonic and seismic responses of offshore oil pipelines in proximity to the seabed using finite element method. Datta and Mashaly analysed the transverse seismic responses of buried [12] and free-spanning [13] submarine pipelines 
under random seismic excitation in the frequency domain based on the spectral approach. Zeinoddini et al. [14] investigated the pipe/water interactions in free-spanning submarine pipelines under severe ground excitations. These studies show that severe earthquakes can result in catastrophic damages to subsea pipelines. How to mitigate these adverse vibrations is deemed important. To the best knowledge of the authors, no open literature reports the vibration control method for subsea pipelines when they are subjected to earthquake loadings.

As will be presented in Section 2, a pipe-in-pipe system can be properly designed as a nonconventional structure-tuned mass damper (TMD) system by adding optimized springs and dashpots in the annulus, which therefore has the potential to mitigate subsea pipeline vibrations induced by various sources without substantially increasing the manufacturing costs and weight of the pipe. A TMD is a device consisting of a mass, a spring and a dashpot that is attached to a vibrating primary structure to attenuate the undesirable vibrations induced by winds or earthquake loadings. The natural frequency of the TMD is tuned to the fundamental vibration frequency of the primary structure so that the damper will resonant out of phase with the original structure and a large amount of the structural vibrating energy is transferred to the TMD and then dissipated by the damper. Due to its simplicity and effectiveness, TMD systems have been widely applied since 1970's in many engineering structures such as tall buildings, towers and bridges [15]. In the conventional TMD design the auxiliary mass is very small, typically in the order of one to a few percent of the primary structure. Due to the small mass of the TMD system, a general agreement on the effectiveness of the conventional TMD system is not formed when it is used to mitigate seismic induced vibrations. Researchers indicate three inherent limitations to the seismic effectiveness of the TMD as summarized by De Angelis et al. [16]: (i) the lack of robustness against deviations in design parameters; (ii) a high dependency on earthquake frequency content; and (iii) the impulsive character of the earthquake excitation. To enhance the effectiveness of the TMD system, a larger mass ratio (up to $100 \%$ and even more in terms of modal quantities) was introduced by some researchers and this system was normally described as a non-conventional TMD [16].

By adding large mass to the primary building and bridge structure is not technically practical and may raise safety issues sometimes. To avoid these problems, the masses already present on the structure to be protected are converted into tuned masses in the nonconventional TMD design, while the structural or architectural function of the structure is retained [16]. In other words, no additional mass is needed for the non-conventional TMD 
system. This non-conventional TMD system has been studied by some researchers recently and was applied in some building (e.g. [17-23]) and bridge [24] structures. Previous studies show that it is feasible and effective to use non-conventional TMD systems to reduce the vibrations of primary structures.

This paper proposes using pipe-in-pipe systems for the vibration control of subsea pipelines. It will be demonstrated that this system can be designed as a non-conventional structureTMD system as mentioned above to mitigate pipeline vibrations. The optimum values for the springs and dashpots installed in the annulus are derived in Section 2. To demonstrate the effectiveness of the proposed system, a subsea pipe-in-pipe system with a free span subjected to transverse earthquake is adopted as an example and numerical analyses are carried out by using the finite element code ANSYS. The detailed numerical modelling is presented in Section 3 and Section 4 defines the earthquake loadings that will be used in the analysis. In section 5, the seismic responses of the traditional and proposed pipe-in-pipe systems are calculated and discussed. Finally in section 6, two possible design options and the robustness of the proposed system are commented.

\section{Pipe-in-pipe as a non-conventional TMD system}

\subsection{Traditional pipe-in-pipe system}

There are two types of pipe-in-pipe systems commonly used in the offshore industry [25]: (i) fully bounded or compliant PIP, in which the entire annulus is filled with insulation material, and (ii) unbounded or non-compliant PIP, in which the insulation is achieved by wrapping standard size insulation pads onto the inner pipe. In the compliant PIP system, load transfer is continuous and the inner and outer pipes deform uniformly. In the non-compliant PIP system the inner and outer pipes can move relative to each other, it is therefore has the potential to be designed as a structure-TMD system and suitable for the vibration control when it is subjected to different sources of vibrations.

Fig. 1 shows a typical non-compliant pipe-in-pipe system. A non-compliant PIP normally comprises an inner pipe, an outer pipe, insulation layer(s), bulkheads and centralizers. Bulkheads are forged fittings attached to the pipe-in-pipe pipeline to maintain structural integrity during installation and operation and to serve as installation aids in variety of ways [1]. They are normally welded to both the inner and outer pipes at several locations especially at both ends, to fully constrain relative axial motions between the inner and outer pipes. The 
centralisers are generally polymeric rings that are clamped on the inner pipe at regular intervals. The spacing between two adjacent centralizers may be 2 meters for reeled pipelines and can up to 12 meters for the S-lay and J-lay installation methods [1]. The purpose of the centralizers is to effectively centralize the inner pipe to prevent possible damage (like abrasion or crushing) to the thermal insulation layer during installation and to minimize loads on the insulation layer during installation and operation. To facilitate the installation of inner pipe and centralizers, a gap of 1 to $10 \mathrm{~mm}$ is usually reserved between the centralizers and the outer pipe [6].

\subsection{Proposed pipe-in-pipe system and equivalent TMD simplification}

By examining the structural layout of the non-compliant pipe-in-pipe system as shown in Fig. 1 and also by comparing it with the structure-TMD concept mentioned in Section 1, it can be seen that the pipe-in-pipe system has the potential to be designed as a nonconventional structure-TMD system by replacing the hard polymeric centralisers by optimized springs and dashpots to connect the inner and outer pipes. By optimizing the spring stiffness and damping coefficient, the inner pipe can vibrate out of phase with the outer pipe and the vibration of the systems therefore can be suppressed. Fig. 2 shows the proposed pipein-pipe system.

Fig. 3 shows the structural model of a typical structure-TMD system. This model consists of a main system and a TMD system. The main system is characterized by the mass $m_{S}$, stiffness $k_{S}$ and damping coefficient $c_{S}$. The corresponding parameters for the TMD system are $m_{T}, k_{T}$ and $c_{T}$ respectively. For the proposed pipe-in-pipe system shown in Fig. 2, the outer pipe can act as the main system and the inner pipe can be considered as the TMD mass. The stiffness and damping of the main system are determined by the surrounding environment (e.g. they are provided by the rock dumping for the unburied pipelines or surrounding soil for the buried pipelines). The optimized springs and dashpots provide stiffness and damping to the TMD system. According to this simplification, the mass ratio between the TMD system and main system is much larger than the conventional TMD configuration. The proposed pipe-in-pipe system should be framed into the non-conventional TMD class as mentioned above.

\subsection{Governing equations}


The equation of motion of the simplified system shown in Fig. 3 subjected to earthquake loading can be written as

$$
\left[\begin{array}{cc}
m_{S} & 0 \\
0 & m_{T}
\end{array}\right]\left\{\begin{array}{l}
\ddot{x}_{S} \\
\ddot{x}_{T}
\end{array}\right\}+\left[\begin{array}{cc}
c_{S}+c_{T} & -c_{T} \\
-c_{T} & c_{T}
\end{array}\right]\left\{\begin{array}{l}
\dot{x}_{S} \\
\dot{x}_{T}
\end{array}\right\}+\left[\begin{array}{cc}
k_{S}+k_{T} & -k_{T} \\
-k_{T} & k_{T}
\end{array}\right]\left\{\begin{array}{l}
x_{S} \\
x_{T}
\end{array}\right\}=-\left\{\begin{array}{l}
m_{S} \\
m_{T}
\end{array}\right\} \ddot{x}_{g}
$$

where $x_{S}$ and $x_{T}$ are the relative displacement of the main and TMD systems with respect to the ground and $\ddot{x}_{g}$ is the ground acceleration. The overdots indicate differentiation with respect to time $t$.

It is convenient to define the uncoupled vibration frequencies and viscous damping ratios of the main and TMD systems as

$$
\begin{gathered}
\omega_{S}=\sqrt{k_{S} / m_{S}}, \xi_{S}=c_{S} / 2 m_{S} \omega_{S} \\
\omega_{T}=\sqrt{k_{T} / m_{T}}, \xi_{T}=c_{T} / 2 m_{T} \omega_{T}
\end{gathered}
$$

and the mass ratio and tunning frequency as

$$
\begin{aligned}
& \mu=m_{T} / m_{S} \\
& \gamma=\omega_{T} / \omega_{S}
\end{aligned}
$$

Numerous methods (e.g. [16, 17], [20], [24], [26-30]) have been proposed to estimate the optimum TMD parameters after the pioneering work done by Den Hartog [31]. In the present study, the optimum formulas proposed by Sadek et al. are adopted due to their effectiveness for the vibration control [27], in which the proposed optimum tunning frequency $\gamma_{o p t}$ and damping ratio $\xi_{\text {opt }}$ are expressed as [27]

$$
\begin{gathered}
\gamma_{o p t}=\frac{1}{1+\mu}\left(1-\xi_{S} \sqrt{\frac{\mu}{1+\mu}}\right) \\
\xi_{o p t}=\frac{\xi_{S}}{1+\mu}+\sqrt{\frac{\mu}{1+\mu}}
\end{gathered}
$$

The optimum stiffness and damping of the TMD system thus can be calculated as

$$
\begin{gathered}
k_{T, o p t}=m_{T} \omega_{S}^{2} \gamma_{o p t}^{2} \\
c_{T, o p t}=2 \xi_{o p t} \sqrt{m_{T} k_{T, o p t}}
\end{gathered}
$$




\section{Numerical modelling}

\subsection{Subsea pipe-in-pipe systems}

Fig. 4 shows a buried subsea pipeline system, a free span is formed due to the unevenness and scouring of the seabed. To mitigate the possible vibrations of the free span induced by vortex shedding or earthquake, the proposed pipe-in-pipe system shown in Fig. 2 is used. Table 1 gives the geometric properties of the steel inner and outer pipes. The length of the free span is $L=30 \mathrm{~m}$. To minimize the influence of the boundary conditions, the shoulder lengths are taken as three times of the free span [32], i.e. $L_{\text {shoulder }}=3 L=90 \mathrm{~m}$. The total length of the analysed pipe-in-pipe system is therefore $210 \mathrm{~m}$. The two ends of the inner and outer pipes are rigidly connected by bulkheads.

The interaction between the soil and the pipeline shoulders are considered by the linear elastic soil springs as suggested by DNV-RP-F105 [33]. The spring stiffness in the lateral $\left(K_{L}\right)$, vertical $\left(K_{V}\right)$ and axial $\left(K_{A}\right)$ directions is given in Table 2 [34]. These parameters correspond to a soil condition of loose sand [34].

\subsection{Numerical models}

Three-dimensional (3D) finite element (FE) model of the proposed pipe-in-pipe system is developed by using finite element code ANSYS. Both the inner and outer pipes are modelled by SHELL63 element, an elastic shell with six degrees of freedom at each node. The material properties of the steel inner and outer pipes are shown in Table 3. The cross sections of the inner and outer pipes are divided into 24 elements as suggested by Saberi et al. [35]. In the axial direction of the pipeline, the element size should be in the order of the outer diameter of the pipeline according to the recommendation given by DNV-RP-F105 [33]. Therefore an element size of $0.3 \mathrm{~m}$ is used in the axial direction.

For the free span of the pipeline, the interaction between the free span and the surrounding water should be taken into account. The effective mass $M_{e}$ of the free span can be calculated as $[11,13]$

$$
M_{e}=M_{p}+M_{a}-M_{b}
$$

where $M_{p}$ is the structural physical mass, which is provided by the free span outer pipe; $M_{b}$ denotes the buoyant mass, which is the mass of the water displaced by the pipe; and $M_{a}$ is the 
added mass, which arises from the fact that the submerged body can impart an acceleration to some of the surrounding fluid. $M_{a}$ can be calculated as [1]

$$
M_{a}=C_{m} \cdot \frac{\pi}{4} D^{2} \cdot \rho_{\text {water }}=C_{m} M_{b}
$$

where $D$ is the outer diameter of the pipe as shown in Fig. 4, which is $0.324 \mathrm{~m}$ in the present study; $\rho_{\text {water }}=1030 \mathrm{~kg} / \mathrm{m}^{3}$ is the seawater density; and $C_{m}$ is the inertia coefficient. $C_{m}$ is related to the proximity of the pipe to the seabed, represented by the ratio $d / D$, where $d$ is the clearance between pipeline and seabed (see Fig. 4 ). For a cylinder, $C_{m}$ can vary exponentially from 1.0 (for $d / D=$ infinity) to 2.29 (for $d / D=0$ ) [11]. In the present study, $C_{m}=1.13$ is assumed [11]. To model the added mass, MASS21 element, a point element having up to six degrees of freedom in ANSYS is used and each node at the free span of the outer pipe is attached with one added mass. For the pipelines in the shoulder and the inner pipe, only the physical masses are considered since they are either buried in the soil or protected from the water by the outer pipe.

With the simplifications mentioned above, the total mass of the outer and inner pipes shown in Fig. 4 can be calculated, with $M_{S}=19598 \mathrm{~kg}$ and $M_{T}=16714 \mathrm{~kg}$ respectively. The mass ratio $\mu$ defined in Eq. (4) reaches $85.3 \%$, which is much larger than the conventional TMD mass ratio.

The interaction between the soil and the pipeline shoulders are considered by the linear elastic soil springs and they are modelled by COMBIN14 elements along the pipe shoulder with an interval of pipeline element size in the axil direction $(0.3 \mathrm{~m})$. At the cross section, these soil springs are extending in three perpendicular directions with respect to the pipe. One end of the soil spring is rigidly connected with a pipe node and the other end is fixed. Fig. 5 shows the distribution of the soil springs around the cross section of the outer pipe. It is noted that in the numerical model, the contribution of each transverse/vertical spring to the total lateral/vertical stiffness is proportional to its share of the perimeter when projected onto the diameter [35]. It results in that the lateral/vertical springs located at the centre of the cross section are the stiffest. In the axial direction, the contribution of each spring is assumed to be the same.

The vibration frequencies and modes of the outer pipe can be calculated by carrying out an eigenvalue analysis after soil spring stiffness is determined. It is found that the first vibration mode is in the transverse direction with a frequency of $1.7733 \mathrm{~Hz}$, the circular vibration 
frequency is thus $\omega_{S}=11.142 \mathrm{rad} / \mathrm{s}$. Fig. 6 shows the fundamental vibration mode of the outer pipe, in which only the parts that are close to the free span are shown.

The damping of the outer pipe is normally considered to comprise hydrodynamic damping, soil damping and structural damping, which account for the contributions of the surrounding water, supporting soil and structure itself to the overall damping ratio. In the present study, a total damping ratio of $\xi_{S}=5 \%$ is assumed $[11,13]$ and modelled by COMBIN14 elements in ANSYS.

With all the parameters defined above, it is able to estimate the optimum parameters for the simplified TMD system. Table 4 tabulates the calculated values by using the formulas proposed by Sadek et al. [27].

To demonstrate the effectiveness of the proposed pipe-in-pipe system to mitigate seismic induced vibrations, only the transverse earthquake loading ( $x$ direction as shown in Figs. 4 and 5) is considered in the present study. The springs and dashpots are installed both in the $+x$ and $-x$ directions with a spacing of $3 \mathrm{~m}$ along the pipe axis. The total number of springs and dashpots is 138 for the analysed pipe-in-pipe system. For each spring and dashpot, the stiffness and damping coefficient are therefore $k_{1}=k_{T, o p t} / 138=4087 \mathrm{~N} / \mathrm{m}$ and $c_{1}=$ $c_{T, \text { opt }} / 138=993 \mathrm{Ns} / \mathrm{m}$, respectively.

These connecting springs are modelled by COMBIN39 elements in ANSYS, in which userdefined force-displacement relationship can be used. In the present study, a bi-linear forcedisplacement relationship is assumed and shown in Fig. 7(a), where $\delta$ is assumed to be the size of the annulus and it is $0.0405 \mathrm{~m}$ according to Table I. When the relative displacement between the inner and outer pipes is smaller than $\delta$, the stiffness is the optimum value $\mathrm{k}_{1}$. On the other hand, when the relative displacement is larger than $\delta$, the spring becomes quite hard to be compressed and a large stiffness $\mathrm{k}_{2}$ is defined. A very large $\mathrm{k}_{2}$ can result in the simulation difficult to converge, a $\mathrm{k}_{2}=4 \times 10^{6} \mathrm{~N} / \mathrm{m}$ is found to have a good balance between the effectiveness and efficiency and is used in the simulations. COMBIN14 elements are used to simulate the dashpots and the damping coefficient is set as $\mathrm{c}_{1}$. It should be noted that a bi-linear force-displacement relationship is assumed for the spring and $\delta$ is assumed as the annulus size in the present study for simplicity. In the actual design, the forcedisplacement relationship can be more accurately obtained when the material or device used to connecting the inner and outer pipes is selected. 
For comparison, the seismic responses of the traditional pipe-in-pipe system shown in Fig. 1 are also calculated. The inner and outer pipes are similarly modelled as described above. In this traditional system, a gap of $5 \mathrm{~mm}$ is assumed between the centralizer and the outer pipe. Colliding between the centralizer and the outer pipe may take place during an earthquake due to the existence of the gap. To consider the possible pounding phenomena, an impact element is adopted and modelled by the COMBIN40 element in ANSYS. Fig. 8 shows a typical impact element, it includes a gap element, a spring and a dashpot. The spring and dashpot will be activated when the gap is closed. Fig. 7(b) shows the force-displacement relation of the impact element, where $\Delta$ is the gap size.

The impact stiffness $\left(k_{p}\right)$ and damping coefficient $\left(c_{p}\right)$ are two parameters that need be determined in the impact element. No research has been carried out on the pounding effect between two concentric pipes, the concept used in the bridge or building poundings is adopted herein. Previous studies (e.g. [36, 37]) suggested that $k_{p}$ can be selected as 10 to 40 times of the lateral stiffness of the stiffer adjacent structures. In the present study, the outer pipe is stiffer than the inner pipe and the stiffness of outer pipe can be calculated as $2.43 \times$ $10^{6} \mathrm{~N} / \mathrm{m}$ based on the mass and vibration frequency calculated above. The total pounding stiffness of $5 \times 10^{7} \mathrm{~N} / \mathrm{m}$ is used in the simulation, which is about 20 times of the outer pipe stiffness. For each impact element, the impact stiffness is thus $k_{p}=5 \times 10^{7} / 138=3.62 \times$ $10^{5} \mathrm{~N} / \mathrm{m}$.

The damping constant determines the energy dissipated during impacts, it can be determined by relating it to the coefficient of restitution $(e)$ at pounding [38], the total damping coefficient can be expresses as

$$
c_{p, \text { total }}=2 \xi_{p} \sqrt{k_{p} \frac{m_{S} m_{T}}{m_{S}+m_{T}}}
$$

with

$$
\xi_{p}=\frac{-\ln e}{\sqrt{\pi^{2}+(\ln e)^{2}}}
$$

The restitution coefficient $e$ is related to the material of two colliding bodies and priorimpact velocity, and it is normally within the range of 0.4 and 0.8 based on the experimental study carried out by Jankowski [39]. To the best knowledge of the authors, there is no open literature reports the pounding between steel (outer pipe) and polymeric material 
(centralizer), $e=0.5$ is assumed in the present study. According to Eqs. (12) and (13), $\xi_{p}$ and $c_{p, \text { total }}$ can be calculated as 0.215 and $4.08 \times 10^{5} \mathrm{Ns} / \mathrm{m}$. $c_{p}$ thus equals $c_{p, \text { total }} / 138=$ $2956 \mathrm{Ns} / \mathrm{m}$.

It is noted that the selection of the impact element parameters might be a bit arbitrary in the present study due to a lack of relevant studies and therefore understanding of the impact between two pipes. However, many previous studies show that pounding between two adjacent structures mainly results in local damage around the pounding location, its influence on the global response is not evident. In the present study, the global displacement response is of interest, which is not significantly affected by the impact element parameters.

\section{Earthquake loadings}

Pipe-in-pipe systems are normally located in the subsea, earthquake time histories at the seafloor should be selected as the inputs. However, most of previous earthquake time histories are recorded at offshore sites, the seafloor recordings are very limited. Moreover, only the transverse input is considered in the present study, this horizontal out-of-plane motion is resulted from the $\mathrm{SH}$ wave, and it is not affected by the upper seawater since water is generally regarded as ideal fluid and cannot transmit shear waves [40]. Therefore earthquake ground motions recorded at onshore sites are used as inputs in the present study.

Three different earthquake loadings are considered in the present study. The first one is an artificially simulated earthquake ground motion based on the spectral representation method recently proposed by the authors [41]. This earthquake ground motion time history is generated to be compatible with the design spectrum for soft soil site (class De) specified in the Australian seismic design code AS1170.4 [42]. In the simulation, the peak ground acceleration (PGA) is set as $0.2 \mathrm{~g}$ and time duration is $20 \mathrm{sec}$, the sampling frequency and upper cut off frequency are 100 and $25 \mathrm{~Hz}$, respectively. Fig. 9(a) shows the simulated acceleration time history and Fig. 10 compares the response spectra of the generated time history and the given model, good match is observed. The second time history is recorded during the 1989 Loma Prieta earthquake. This is a near-field ground motion characterized with long-period pulse-like waveforms as shown in Fig. 9(b). The last record is from the 1940 El Centro earthquake. It is used to represent the far-field earthquake ground motion. The acceleration time history of this earthquake is shown in Fig. 9(c). Table 5 summaries the information of the ground motions used in the analysis. 


\section{Numerical results}

The free span vibration of the subsea pipeline belongs to a general class of structure-water interaction problem. It is important to correctly assess the reactive force generated between the pipe and the surrounding water during vibration. This reactive force is mainly due to the inertia and pressure drag effects. The inertia effect is considered by the added mass as mentioned in Section 3.2. The transverse drag force per unit length of the pipeline can be express as [11]

$$
F_{D}=\frac{1}{2} \rho_{\text {water }} C_{D} D v_{n}\left|v_{n}\right|
$$

where $C_{D}$ is the transverse drag coefficient and $v_{n}$ is the transverse water particle velocity. In the case of seismic excitation, Nath and Soh [11] found that the drag effect does not appear to be substantial and its effect is mainly noticeable at response peaks. Not to further complicate the problem, drag effect is neglected in the present study and only the seismic loading is considered as input in the numerical simulation.

To investigate the effectiveness of the proposed pipe-in-pipe system to mitigate seismic induced vibrations of a subsea pipeline with a free span, the seismic responses of the proposed pipe-in-pipe system subjected to the three different earthquake loadings as given above are investigated. For comparison, the seismic responses of the traditional pipe-in-pipe system are also calculated.

Fig. 11 shows the transverse displacement time histories of the outer and inner pipes at the middle of the free span when the proposed and traditional pipe-in-pipe systems are subjected to different earthquake loadings. The blue curves are the results obtained based on the traditional PIP model and the red curves are from the proposed system. As can be seen from the figure, it is quite effective to use the proposed pipe-in-pipe system to mitigate seismic induced vibrations of the free span. The proposed system not only significantly suppresses the vibration of the main system (outer pipe) but also obviously reduces the vibration of the TMD system (inner pipe). Table 6 tabulates the peak responses. The corresponding ratios between the responses of the proposed system and the traditional system are also given in the table. As shown, the ratios of the outer pipe are $0.424,0.500$ and 0.541 respectively for the three earthquake loadings with an average of 0.488 . For the inner pipe, the values are 0.528 , 0.565 and 0.570 respectively and the average is 0.554 . These results demonstrate that properly designing a pipe-in-pipe system can greatly reduce pipeline vibrations. The 
proposed system is more effective in reducing the vibration of the outer pipe compared to the inner pipe. This is because the optimum values of the TMD system are estimated based on the structure-TMD concept, in which normally only the vibrations of the main system is of interest. For the proposed system, due to the large mass ratio, the vibrations of both the outer and inner pipes are evidently suppressed. This is, actually, a very favourable property for the proposed pipe-in-pipe system, since as mentioned above, the inner pipe is used to transport the hydrocarbons, the safety of the inner pipe is as important as the outer pipe. These results also show that almost the same level of reductions can be obtained for all the three earthquake loadings, which means that the mitigation effectiveness is independent of the ground motion frequencies. The proposed system is effective for different types of earthquake ground motions.

Fig. 12 shows the relative displacement between the outer and inner pipes at the middle of the free span. The gap size between the centralizer and the outer pipe of the traditional pipein-pipe system ( $5 \mathrm{~mm}$ ) and the size of the annulus of the proposed pipe-in-pipe (40.5 $\mathrm{mm}$ ) are also plotted in this figure by the red dash lines. As shown in the first column of Fig. 12 (traditional PIP system), the movement of the inner pipe is constrained by the outer pipe and the centralizer contacts with the outer pipe several times during the earthquake excitations due to the very small gap size, which makes the outer and inner pipes almost vibrate together (see the blue curves for the same earthquake in Fig. 11). It also can be seen from the figure that the relative displacement between the outer and inner pipes can sometimes slightly larger than the gap size, which means penetrations occur. This is because impact element COMBIN40 is used in the numerical simulation and penetration is allowed by this element. In fact, the pounding force is calculated based on the penetration depth by using this method. For the proposed pipe-in-pipe system as shown in the right column of Fig. 12, there is no intersection between the blue curve and the red dash lines, indicating that the inner pipe can vibrate freely inside the outer pipe and the TMD function can be fully developed, which in turn significantly reduces the vibrations of both the outer and inner pipes.

When more severe earthquake occurs, the relative displacement between the outer and inner pipes can be large and the inner pipe might not be able to oscillate freely inside the outer pipe. To further investigate the effectiveness of the proposed system, the seismic responses of the traditional and proposed systems subjected to the simulated earthquake with a larger PGA of $0.5 \mathrm{~g}$ are also calculated. Figs. 13 and 14 show the transverse and relative displacements at the middle of the free span of the outer and inner pipes, respectively. The peak displacements are 
tabulated in Table 6 as well. As shown in the right column of Fig.14, the vibration of the inner pipe is constrained by the outer pipe, the TMD function cannot be fully developed, which makes the system less effective compared to the less severe earthquake (PGA=0.2g) where the TMD function is fully developed. As shown in Table 6, the ratios are 0.542 and 0.675 respectively for the outer and inner pipes in this case, which are about $28 \%$ larger than the corresponding values from the case of PGA=0.2g. However, broadly speaking the reduction is still quite appealing as shown in Fig. 13.

\section{Possible design options and robustness of the proposed system}

\subsection{Possible design options}

Only the numerical simulations are carried out in the present study and the hard centralizers are replaced by the optimized springs and dashpot in the numerical model. In real practice, many options can be used to connect the inner and outer pipes. The polyurethane foam (PUF) might be one of the options. PUF with different density can provide different stiffness, and therefore the optimum stiffness can be achieved by selecting the PUF with proper density. The damping requirement can be obtained by adjusting the PUF length along the pipe. Moreover, as will be discussed in Section 6.2, the proposed PIP system is quite robust due to the large mass ratio, i.e., the vibration suppression capability is not greatly affected by variations in the stiffness and damping of the connecting spring and dashpot. This property will significantly facilitate the design.

Another option can be the rotational friction hinge device with spring (RFHDS) as shown in Fig. 15. The rotational friction hinge device can provide the optimum damping by adjusting the preload applied on the bolts and the spring can provide the required stiffness. In real practice, one end of the device can be welded to the inner pipe, while a small gap will be reserved between the other end and the outer pipe to facilitate installation. The device will be activated when the outer pipe contacts with it.

Both these two options will not change too much of the traditional design and are not difficult to be applied in the annulus, they will not increase too much of the cost and therefore have great application potentials. Some prototype models will be fabricated and laboratory tests will be performed in the next step.

\subsection{Robustness of the proposed system}


There are always certain uncertainties exist in the subsea pipe-in-pipe systems. For example, it is quite difficult to exactly determine the property of the surrounding soil and the length of the free span may keep changing during operation. Moreover, both the stiffness and damping of the TMD system should be optimized to effectively mitigate the vibration of the system. These, however, might not be easily satisfied in the design to accommodate the constantly changing PIP and loading conditions. Furthermore, only the earthquake induced vibration is investigated in the present study, vortex shedding can be another vibration source. Using these optimum values to mitigate vortex shedding induced vibrations might not be as effective as they are in mitigating earthquake ground motion induced vibrations. Due to these reasons, the robustness of the system is therefore important. To demonstrate the robustness of the proposed pipe-in-pipe system for the vibration control, the simplified structure-TMD system shown in Fig. 3 is further investigated. The mean square response of the main system can be defined as $[24,26]$

$$
E\left[x_{S}^{2}\right]=\int_{-\infty}^{\infty}\left|H_{S}(\omega)\right|^{2} S(\omega) d \omega
$$

in which $S(\omega)$ denotes the spectral density of the ground excitation. For simplicity, whitenoise excitation is normally assumed [17, 26] and Eq. (15) can be further simplified as

$$
E\left[x_{S}^{2}\right]=\int_{-\infty}^{\infty}\left|H_{S}(\omega)\right|^{2} S(\omega) d \omega=S_{0} \int_{-\infty}^{\infty}\left|H_{S}(\omega)\right|^{2} d \omega
$$

where $S_{0}$ is the white noise intensity. $H_{S}$ is the transfer function and can be expressed as [24]

$$
H_{S}(\omega)=\frac{m_{S}+Z(\omega)}{m_{S}\left(\omega_{S}^{2}-\omega^{2}+2 i \xi_{S} \omega_{S} \omega\right)-\omega^{2} Z(\omega)}
$$

with

$$
Z(\omega)=\frac{m_{T}\left(\omega_{T}^{2}+2 i \xi_{T} \omega_{T} \omega\right)}{\omega_{T}^{2}-\omega^{2}+2 i \xi_{T} \omega_{T} \omega}
$$

Fig. 16 shows the mean square displacement responses of a main system with different TMD masses subjected to the white-noise earthquake loading of unit spectral intensity as a function of the tunning frequency $\gamma$ and damping ratio $\xi_{T}$. As shown in Fig. 16(a), $E\left[x_{S}^{2}\right]$ is rather flat around the minimum point for the proposed pipe-in-pipe system, which indicates the system will be effective even if the parameters shift away from the optimum values. This characteristic will greatly facilitate the system design. For the conventional TMD system with 
$\mu=1 \%$, Fig. 16(b) shows very steep variation around the minimum value, indicating the optimum values can vary only within a very narrow range in order to achieve the effectiveness of the system.

\section{Conclusions}

This paper proposes using pipe-in-pipe systems for the vibration control of subsea pipelines. This system takes advantage of the special structural layout of the pipe-in-pipe systems and can be designed as a non-conventional structure-TMD system. The outer pipe acts as the main system and the inner pipe performs as the TMD mass. The optimized springs and dashpots are installed in the annulus between the outer and inner pipes and provide stiffness and damping to the TMD mass. To examine the effectiveness of the proposed system to mitigate seismic induced vibrations of a subsea pipeline with a free span, detailed 3D FE analyses are carried out by using finite element code ANSYS. Numerical results show that the proposed system not only significantly suppresses the vibration of the outer pipe but also obviously reduces the vibration of the inner pipe without any additional mass. Moreover, the system is effective and robustness to different types of earthquakes. This system will have great application potentials to control the vibrations of subsea pipelines induced by various sources.

\section{Acknowledgement}

The first author would like to acknowledge the support from Australian Research Council Discovery Early Career Researcher Award DE150100195 for carrying out this research.

\section{References}

[1] Bai Q, Bai Y. Subsea pipeline design, analysis, and installation. Oxford: Gulf Professional Publishing; 2014.

[2] Kyriakides S. Buckle propagation in pipe-in-pipe systems. Part I: experiments. Int J Soilds Struct 2002; 39: 351-66.

[3] Kyriakides S, Vogler TJ. Buckle propagation in pipe-in-pipe systems. Part II: analysis. Int J Soilds Struct 2002; 39: 367-92.

[4] Kyriakides S, Netto TA. On the dynamic propagation and arrest of buckles in pipe-inpipe systems. Int J Soilds Struct 2004; 41: 5463-82.

[5] Gong S, Li G. Buckle propagation of pipe-in-pipe systems under external pressure. Eng Struct 2015; 84: 207-22. 
[6] Wang Z, Chen Z, Liu H. Numerical study on upheaval buckling of pipe-in-pipe systems with full contact imperfections. Eng Struct 2015; 99: 264-71.

[7] O’Rourke MJ, Liu X. Response of buried pipelines subjected to earthquake effects. New York: Multidisciplinary Centre for Earthquake Engineering Research; 1999.

[8] Yang B, Gao FP, Jeng DS, Wu YX. Experimental study of vortex-induced vibrations of a pipeline near an erodible sandy seabed. Ocean Eng 2008; 35: 301-9.

[9] Zhou T, Razali SM, Hao Z, Cheng L. On the study of vortex-induced vibration of a cylinder helical strakes. J Fluid Struct 2011; 27: 903-17.

[10] Kumar RA, Sohn CH, Gowda B. Passive control of vortex-induced vibrations: an overview. Recent Pat Mech Eng 2008; 1: 1-11.

[11] Nath B, Soh CH. Transverse seismic response analysis of offshore pipelines in proximity to the sea-bed. Earthquake Eng Struct Dyn 1978; 6: 569-83.

[12] Datta TK, Mashaly EA. Seismic response of buried submarine pipelines. J Energy Resour Technol 1988; 110: 208-18.

[13] Datta TK, Mashaly EA. Transverse response of offshore pipelines to random ground motion. Earthquake Eng Struct Dyn 1990; 19: 217-28.

[14] Zeinoddini M, Parke GAR, Sadrossadat SM. Free-spanning submarine pipeline response to sever ground excitations: water-pipeline interaction. J Pipeline Syst Eng Pract 2012; 3: 135-49.

[15] Soong TT, Spencer BF. Supplemental energy dissipation: state-of-the-art and state-of-the practice. Eng Struct 2002; 24: 246-59.

[16] De Angelis M, Perno S, Reggio A. Dynamic response and optimal design of structures with large mass ratio TMD. Earthquake Eng Struct Dyn 2012; 41: 41-60.

[17]Feng MQ, Mita A. Vibration control of tall buildings using mega subconfiguration. J Eng Mech, ASCE 1995; 24: 1082-8.

[18] Zhang X, Wang D, Jiang J. The controlling mechanism and the controlling effectiveness of passive mega-sub-controlled frame subjected to random wind loads. J Sound Vib 2005; 283: 543-60.

[19]Ziyaeifar M, Noguchi H. Partial mass isolation in tall buildings. Earthquake Eng Struct Dyn 1998; 27: 49-65.

[20]Reggio A, De Angelis M. Optimal energy-based seismic design of non-conventional tuned mass damper (TMD) implemented via inter-story isolation. Earthquake Eng Struct Dyn 2015; 44: 1623-42. 
[21] Villaverde R. Implementation study of aseismic roof isolation system in 13-story building. J Seismol Earthquake Eng 2000; 2: 17-27.

[22] Villaverde R, Aguirre M, Hamilton C. Aseismic roof isolation system built with steel oval elements: exploratory study. Earthquake Spectra 2005; 21: 225-41.

[23] Tian Z, Qian J, Zhang L. Slide roof system for dynamic response reduction. Earthquake Eng Struct Dyn 2008; 37: 647-58.

[24] Hoang N, Fujino Y, Warnitchai P. Optimal tuned mass damper for seismic applications and practical design formulas. Eng Struct 2008; 30: 707-15.

[25] Van den Abeele F, De Ville Q, Giagmouris T, Onya E, Njuguna, J. Finite element simulation of pipe-in-pipe systems installed on an uneven seabed. Available: $\underline{w w w . s a g e-}$ profile.com/wp-

content/uploads/FE_simulation_of_PIP_systems_with_SAGE_Profile.pdf.

[26] Bakre SV, Jangid RS. Optimum parameters of tuned mass damper for damped main system. Struct Contr Health Monit 2007; 14: 448-70.

[27] Sadek F, Mohraz B, Taylor AW, Chung RM. A method of estimating the parameters of tuned mass dampers for seismic applications. Earthquake Eng Struct Dyn 1997; 26: 61735.

[28] Soong TT, Rana R. Parametric study and simplified design of tuned mass dampers. Eng Struct 1998; 20: 193-204.

[29] Chang CC. Mass dampers and their optimal designs for building vibration control. Eng Struct 1999; 21: 454-63.

[30] Li C, Qu W. Optimum properties of multiple tuned mass dampers for reduction of translational and torsional response of structures subjected to ground acceleration. Eng Struct 2006; 28: 472-94.

[31]Den Hartog JP. Mechanical vibrations. New York: McGraw-Hill; 1956.

[32] Vedeld K, Sollund H, Hellesland J. Free vibrations of free spanning offshore pipelines. Eng Struct 2013; 56: 68-82.

[33]Det Norske Veritas (DNV). Recommended practice DNV-RP-F105: free spanning pipelines. Oslo: DNV; 2006.

[34] Sollund HA, Vedeld K, Hellesland J, Fyrileiv O. Dynamic response of multi-span offshore pipelines. Mar Struct 2014; 39: 174-97.

[35] Saberi M, Behnamfar F, Vafaeian M. A semi-analytical model for estimating seismic behaviour of buried steel pipes at bend point under propagating waves. B Earthquake Eng 2013; 11: 1373-1402. 
[36] Haо H, Ma G. An investigation of the coupled torsional-pounding response of adjacent asymmetrical structures. In: Proceedings of the $7^{\text {th }}$ east-pacific conference on the structural engineering and constructions, Kochi, Japan; 1999. p. 293-310.

[37] Bi K, Hao H, Chouw N. 3D FEM analysis of pounding response of bridge structures at a canyon site to spatially varying ground motions. Adv Struct Eng 2013; 16: 619-40.

[38]Jankowski R, Wilde K, Fujino, Y. Pounding of superstructure segments in isolated elevated bridge during earthquakes. Earthquake Eng Struct Dyn 1998; 27: 487-502.

[39] Jankowski R. Experimental study on earthquake-induced pounding between structural elements made of different building materials. Earthquake Eng Struct Dyn 2010; 39: 343-54.

[40] Li C, Hao H, Li H, Bi K. Theoretical modelling and numerical simulation of seismic motions at seafloor. Soil Dyn Earthquake Eng 2015; 77: 220-5.

[41] Bi K, Hao H. Modelling and simulation of spatially varying earthquake ground motions at sites with varying conditions. Probab Eng Mech 2012; 29: 92-104.

[42] AS 1170.4. Structural design actions-part 5: earthquake actions in Australia. Sydney: Standards Australian; 2007. 
Table 1. Geometric properties of the pipe-in-pipe system

\begin{tabular}{|c|c|c|}
\hline Parameters & Outer pipe & Inner pipe \\
\hline Outer diameter $(\mathrm{m})$ & 0.324 & 0.219 \\
\hline Thickness $(\mathrm{m})$ & 0.012 & 0.016 \\
\hline
\end{tabular}

Table 2. Soil spring stiffness [31]

\begin{tabular}{|c|c|c|}
\hline$K_{L}\left(\mathrm{kN} / \mathrm{m}^{2}\right)$ & $K_{V}\left(\mathrm{kN} / \mathrm{m}^{2}\right)$ & $K_{A}\left(\mathrm{kN} / \mathrm{m}^{2}\right)$ \\
\hline 10944 & 14550 & 10944 \\
\hline
\end{tabular}

Table 3. Material properties of steel inner and outer pipes

\begin{tabular}{|c|c|c|}
\hline Density $\left(\mathrm{kg} / \mathrm{m}^{3}\right)$ & Young's modulus (MPa) & Poisson's ratio \\
\hline 7800 & 210 & 0.3 \\
\hline
\end{tabular}

Table 4. Optimum values of the simplified TMD system

\begin{tabular}{|c|c|c|c|}
\hline$\gamma_{\text {opt }}$ & $\xi_{\text {opt }}$ & $k_{T, o p t}(\mathrm{~N} / \mathrm{m})$ & $c_{T, o p t}(\mathrm{Ns} / \mathrm{m})$ \\
\hline 0.5214 & 0.7054 & $5.640 \times 10^{5}$ & $1.3699 \times 10^{5}$ \\
\hline
\end{tabular}

Table 5. Information of the selected earthquake recordings

\begin{tabular}{|c|c|c|c|c|c|}
\hline Record & Earthquake & Station & Date & PGA (g) & Type \\
\hline G01000 & Loma Prieta & Gilroy Array \#1 & $10 / 18 / 1989$ & 0.4153 & Near-field \\
\hline ELC180 & Imperial Valley & El Centro Array & $05 / 19 / 1940$ & 0.2808 & Far-field \\
\hline
\end{tabular}

Table 6. Peak displacements of the outer and inner pipes at the middle of the free span of the pipe-in-pipe systems and corresponding response ratios

\begin{tabular}{|c|c|c|c|c|c|c|}
\hline \multirow{2}{*}{ Earthquake } & \multicolumn{2}{|c|}{ Traditional PIP $(\mathrm{m})$} & \multicolumn{2}{c|}{ Proposed PIP $(\mathrm{m})$} & \multicolumn{2}{c|}{ Ratio } \\
\cline { 2 - 7 } & Outer & Inner & Outer & Inner & Outer & Inner \\
\hline Simulated-0.2g & 0.118 & 0.123 & 0.050 & 0.065 & 0.424 & 0.528 \\
\hline Loma Prieta & 0.058 & 0.062 & 0.029 & 0.035 & 0.500 & 0.565 \\
\hline El Centro & 0.098 & 0.100 & 0.053 & 0.057 & 0.541 & 0.570 \\
\hline Simulated-0.5g & 0.277 & 0.280 & 0.150 & 0.189 & 0.542 & 0.675 \\
\hline
\end{tabular}




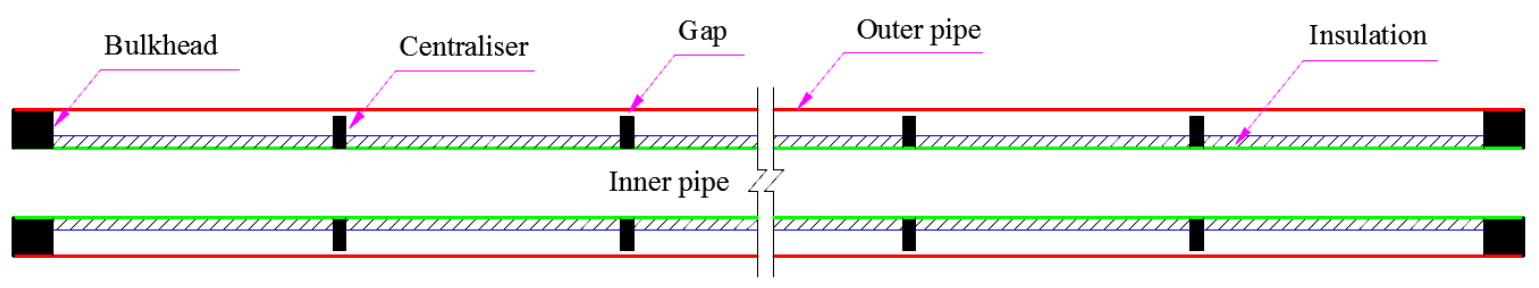

Fig. 1. A typical non-compliant pipe-in-pipe system (not to scale)
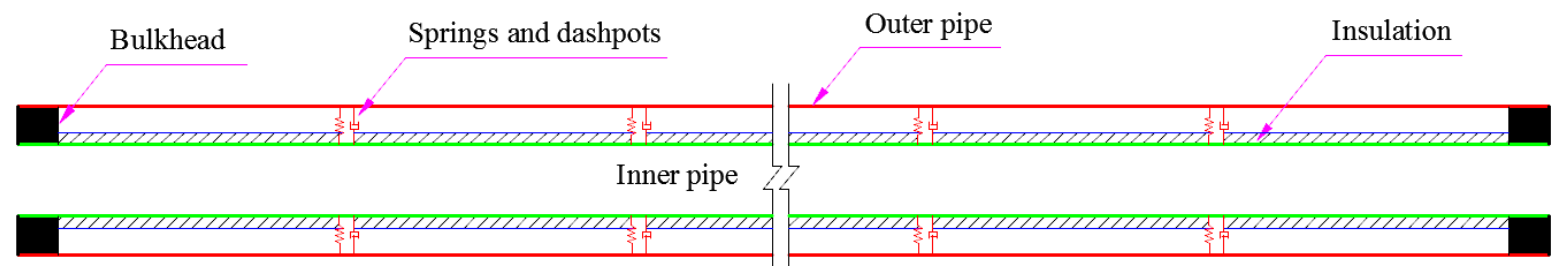

Fig. 2. Proposed pipe-in-pipe system (not to scale)

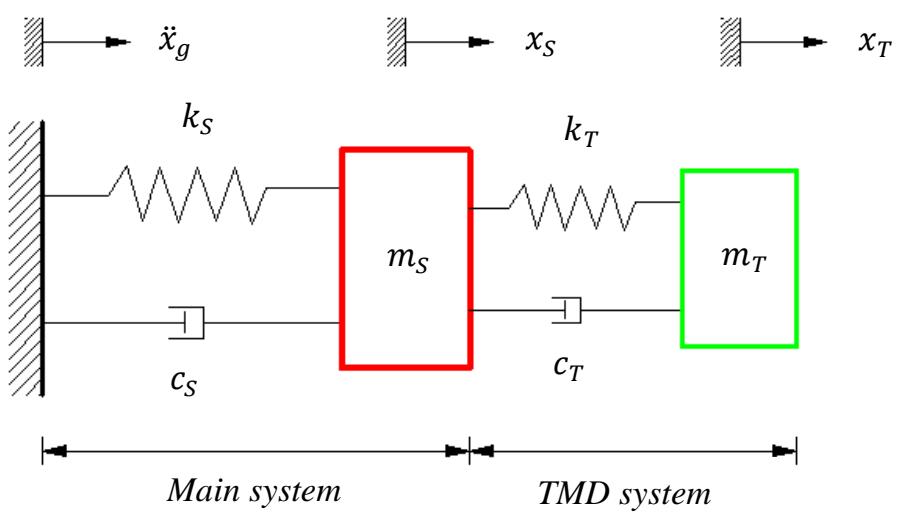

Fig. 3. Structural model of a structure-TMD system subjected to earthquake loading 


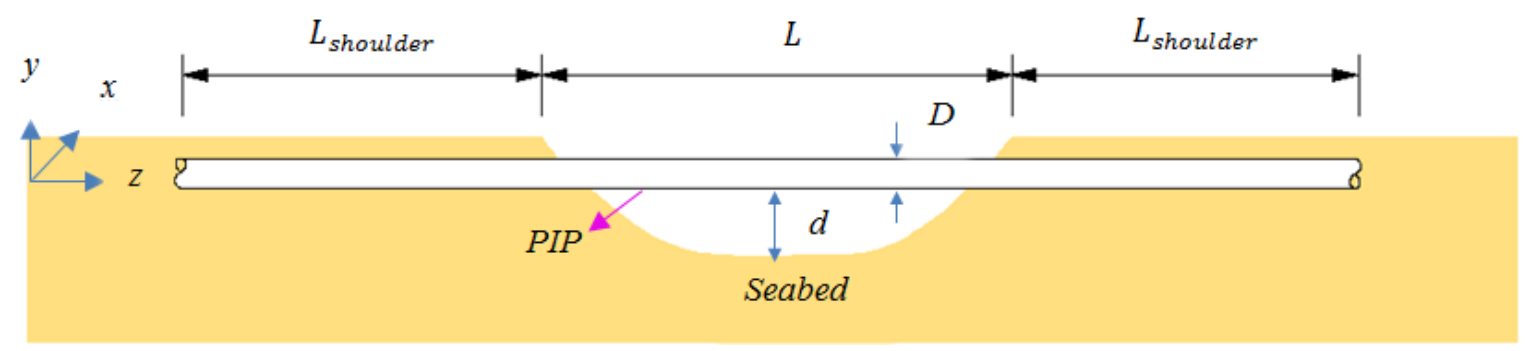

Fig. 4. A subsea pipe-in-pipe system with a free span (not to scale)

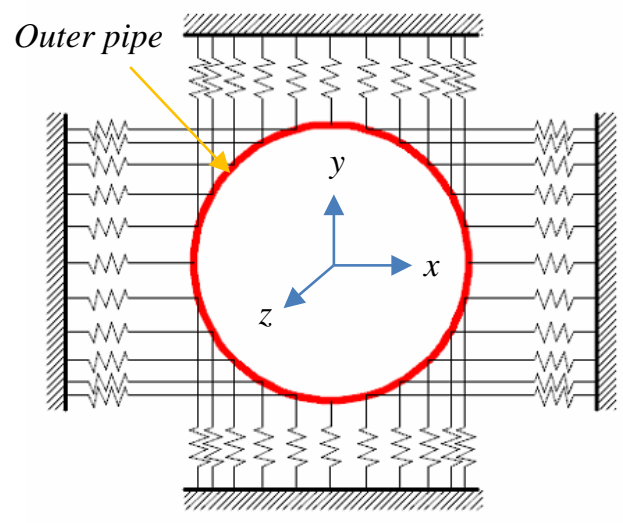

Fig. 5. Distribution of soil springs along the cross section of the outer pipe

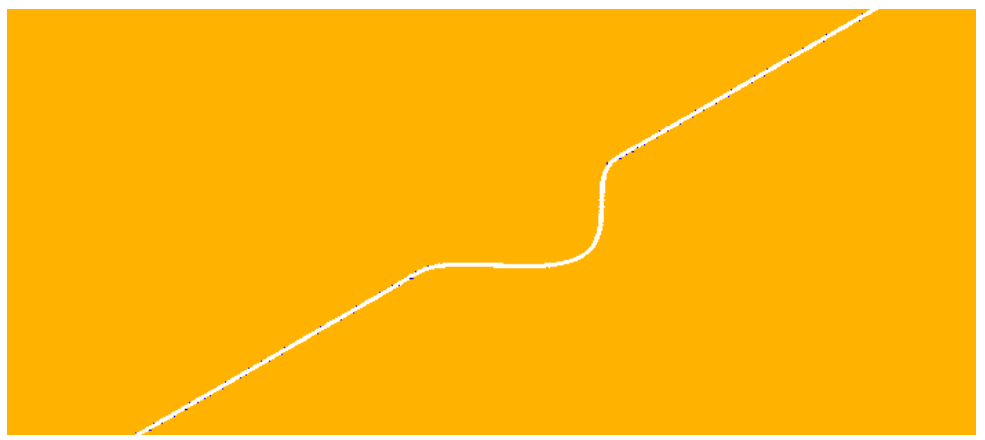

Fig. 6. First vibration mode of the outer pipe 


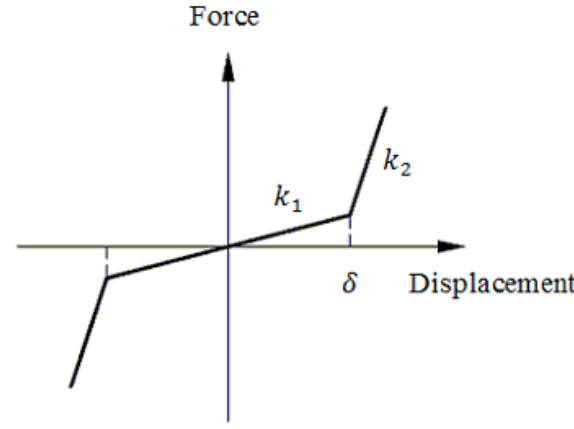

(a)
Impact force

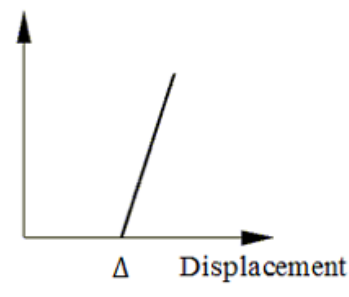

(b)

Fig. 7. Force-displacement relation for (a) connecting spring and (b) impact element

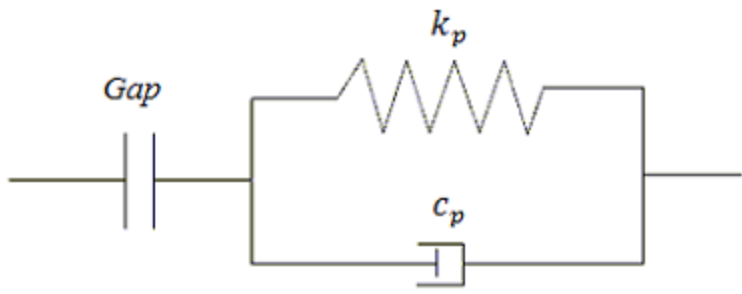

Fig. 8. A typical impact element 
(a)

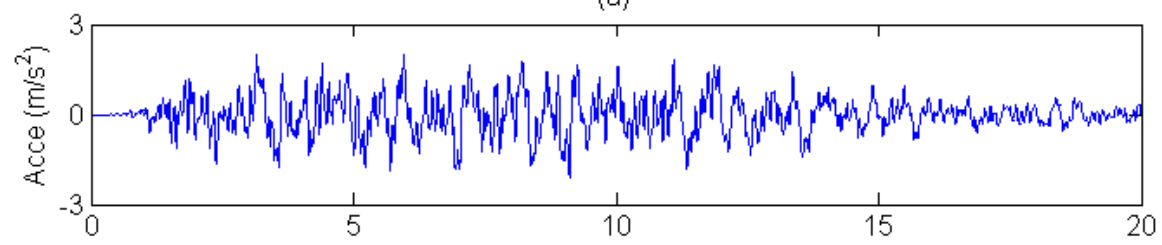

(b)

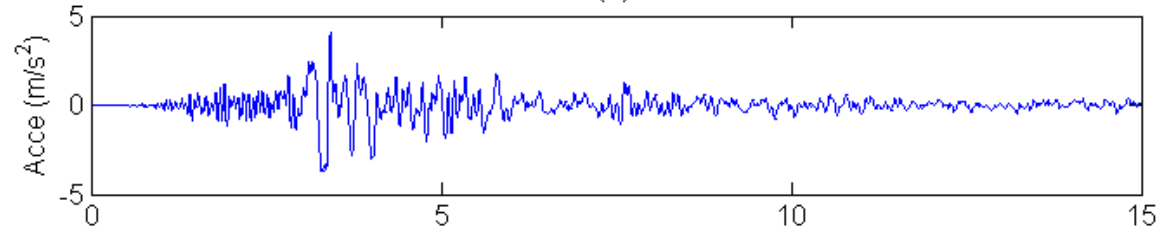

(c)

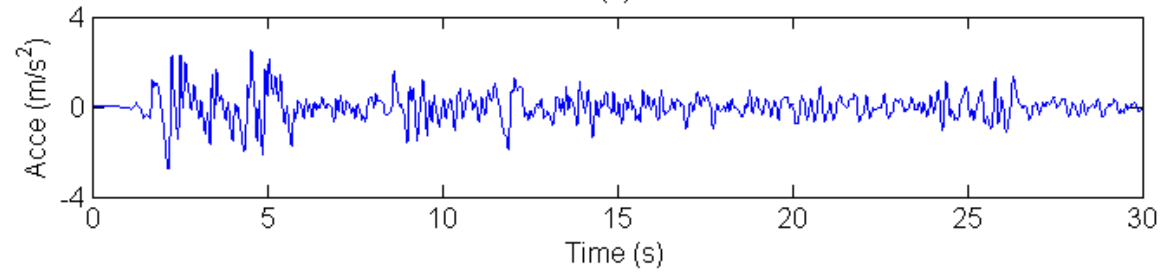

Fig. 9. Earthquake time histories: (a) simulated earthquake; (b) Loma Prieta earthquake; (c) El Centro earthquake

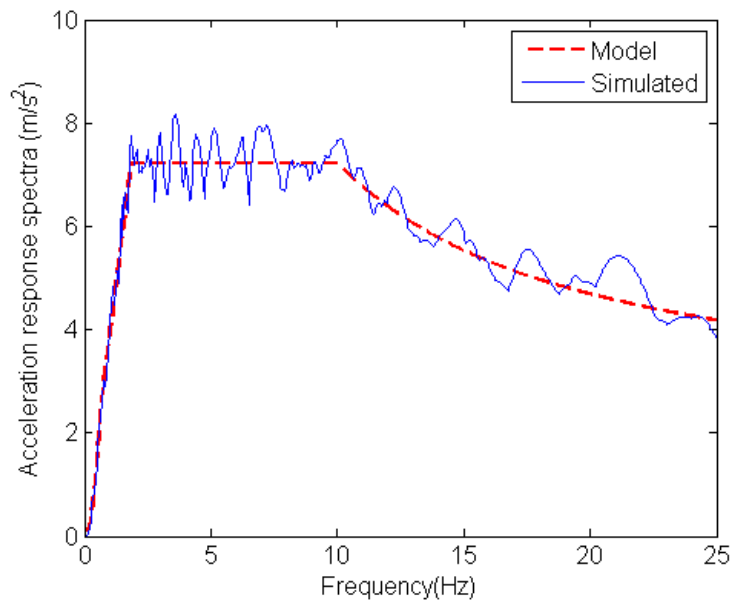

Fig. 10. Comparison of the simulated and target response spectra 

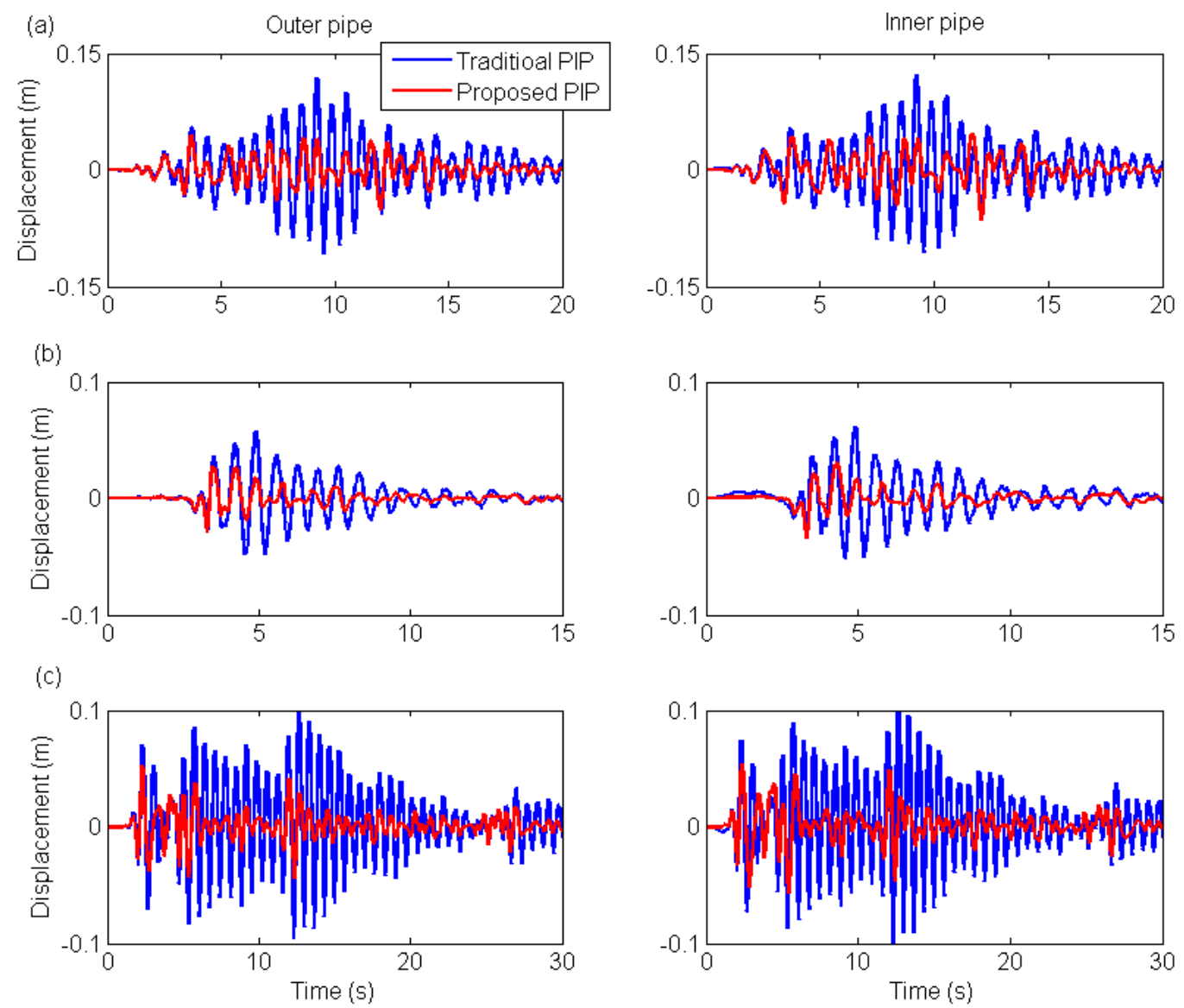

Fig. 11. Transverse displacements of the outer and inner pipes at the middle of the free span when the proposed and traditional pipe-in-pipe systems are subjected to: (a) simulated earthquake; (b) Loma Prieta earthquake; (c) El Centro earthquake 

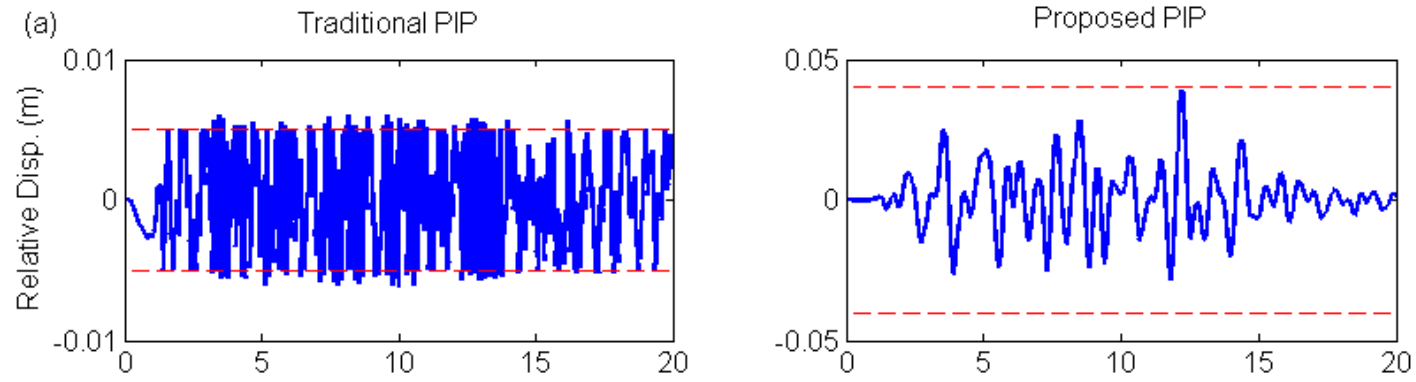

(b)
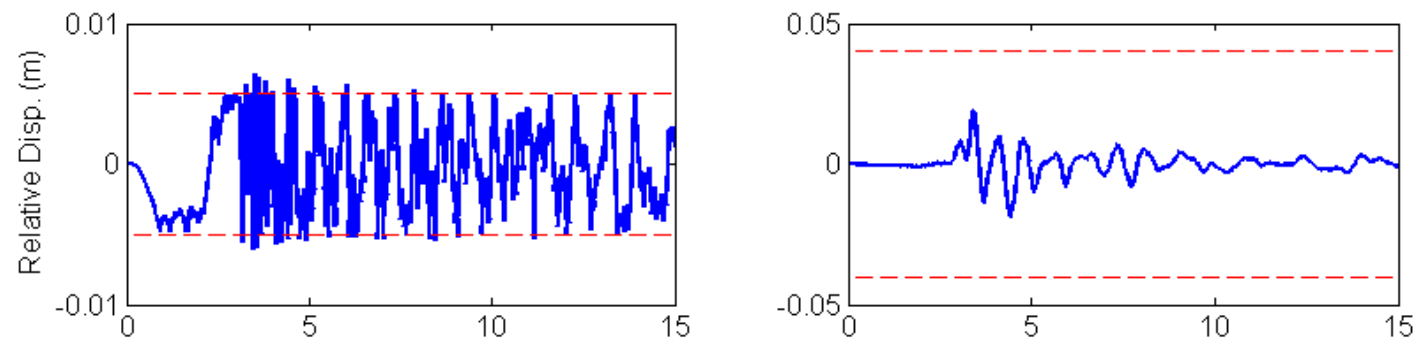

(c)
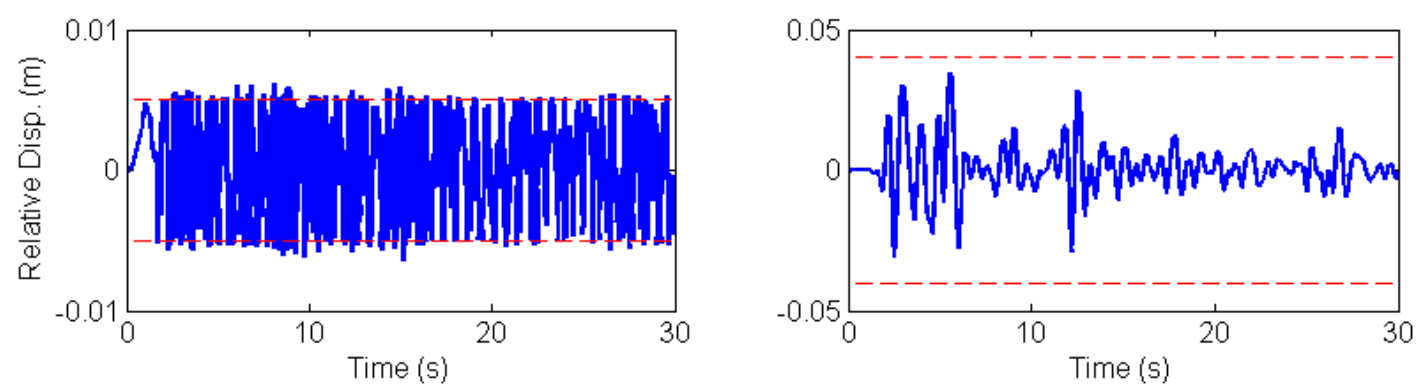

Fig. 12. Relative displacements between the outer and inner pipes at the middle of the free span when the proposed and traditional pipe-in-pipe systems are subjected to: (a) simulated earthquake; (b) Loma Prieta earthquake; (c) El Centro earthquake 


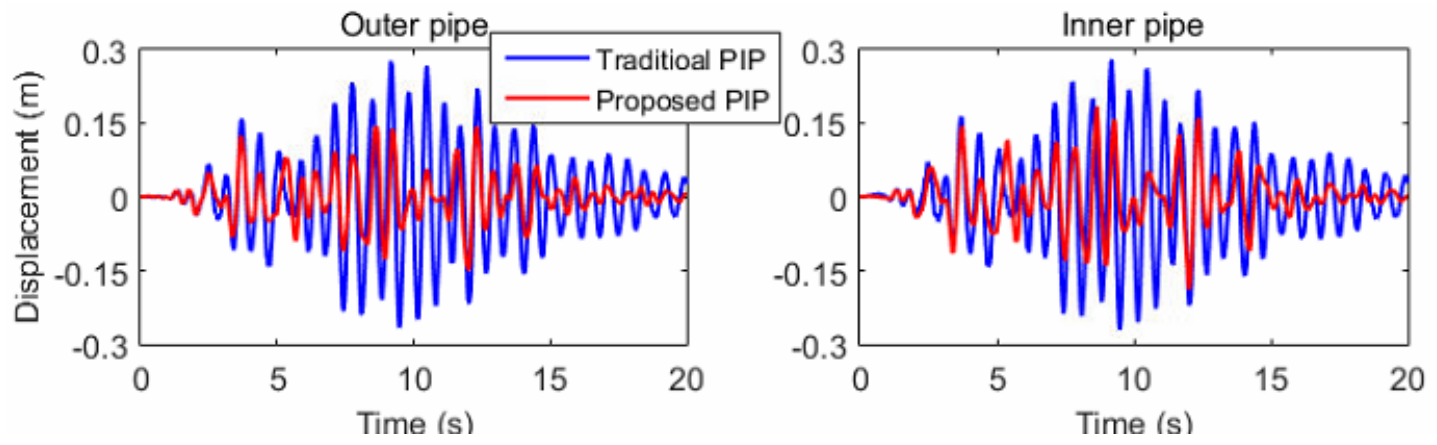

Fig. 13. Transverse displacements of the outer and inner pipes at the middle of the free span when the proposed and traditional pipe-in-pipe systems are subjected to the simulated earthquake with a PGA of $0.5 \mathrm{~g}$
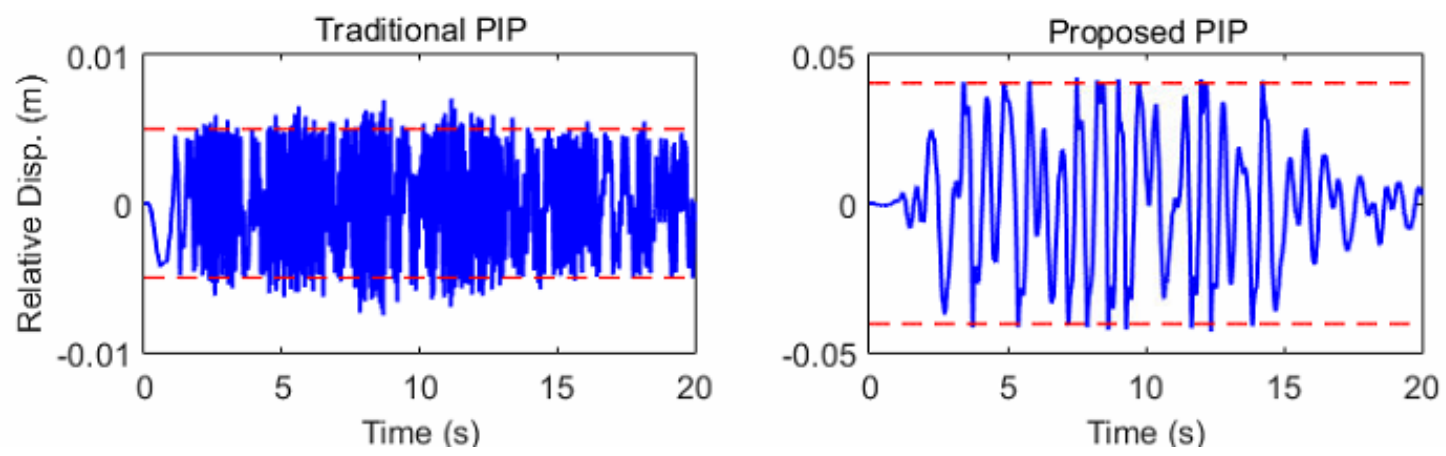

Fig. 14. Relative displacements between the outer and inner pipes at the middle of the free span when the proposed and traditional pipe-in-pipe systems are subjected to the simulated earthquake with a PGA of $0.5 \mathrm{~g}$ 


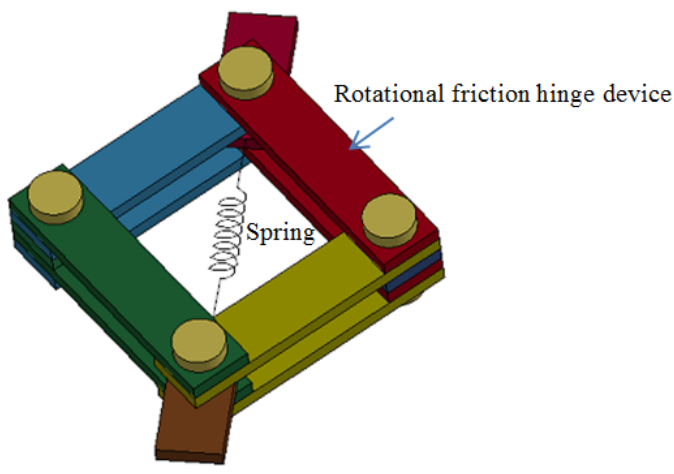

Fig. 15. One possible design option: rotational friction hinge device with spring (RFHDS)

(a)

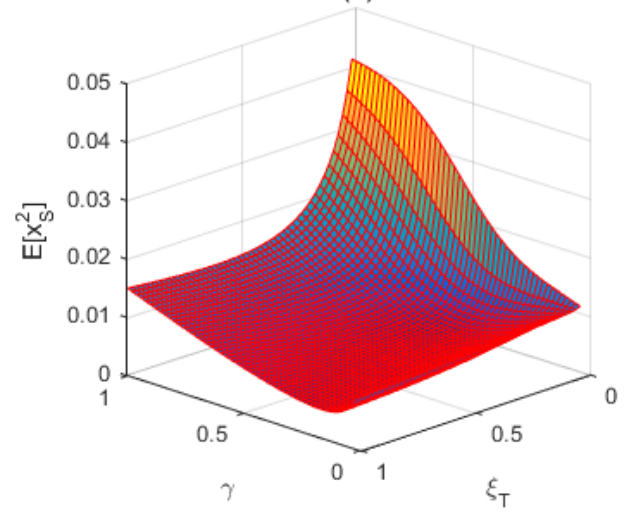

(b)

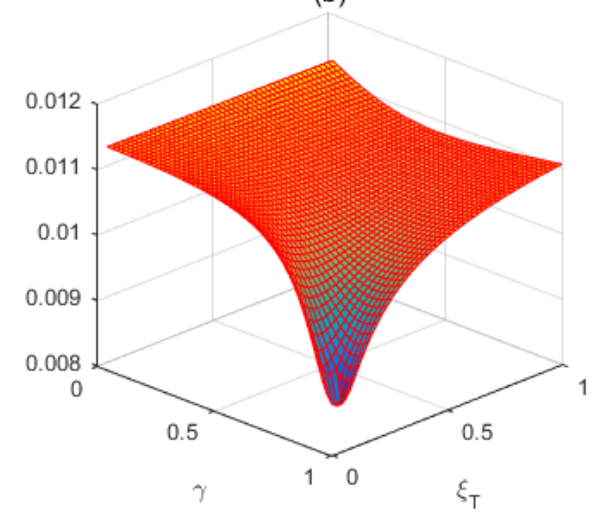

Fig. 16. Mean square displacement responses of a main system with different TMD masses subjected to the white-noise earthquake loading with unit spectral density: (a) $\mu=85.3 \%$, (b)

$$
\mu=1 \%
$$

